\title{
ESPECIAÇÃO DE CROMO EM CIMENTOS E DERIVADOS DE CIMENTO BRASILEIROS
}

\author{
Wladiana Oliveira Matos* \\ Departamento de Química Analítica e Físico-Química, Universidade Federal do Ceará, Fortaleza - Ce, Brasil \\ Joaquim Araújo Nóbrega \\ Departamento de Química, Universidade Federal de São Carlos, CP 676, 13560-960 São Carlos - SP, Brasil
}

Recebido em 7/11/08; aceito em 4/5/09; publicado na web em 30/9/09

\begin{abstract}
CHROMIUM SPECIATION IN CEMENT AND CEMENT-RELATED MATERIALS FROM BRAZIL. A method for determination of $\mathrm{Cr}(\mathrm{III})$ and $\mathrm{Cr}(\mathrm{VI})$ in cement and cement-related materials was studied. Molecular absorption spectrophotometry based on 1,5-diphenylcarbazide as chromogenic reagent was used for determination of $\mathrm{Cr}(\mathrm{VI})$ after alkaline extraction. The total chromium concentration was determined using flame atomic absorption spectrometry (FAAS) after complete sample decomposition by fusion. The quantification of $\mathrm{Cr}(\mathrm{III})$ was accomplished by subtracting the $\mathrm{Cr}(\mathrm{VI})$ concentration from the total chromium concentration. The concentration of $\mathrm{Cr}(\mathrm{III})$ in the samples ranged from 10.9 to $88.0 \mathrm{mg} \mathrm{kg}^{-1}$, whereas only in few samples the $\mathrm{Cr}(\mathrm{VI})$ concentration was higher than the value established by the European Community to this type of sample $\left[2 \mathrm{mg} \mathrm{kg}^{-1} \mathrm{Cr}(\mathrm{VI})\right]$.
\end{abstract}

Keywords: chromium; speciation; cement.

\section{INTRODUÇÃO}

Partículas sólidas podem ter efeitos negativos na qualidade do ar e um dos processos industriais que causam poluição devido à emissão de partículas é a indústria de cimento. Embora indústrias de cimento sejam geralmente instaladas distante dos centros urbanos, as áreas próximas às suas instalações são afetadas, pois pós de cimento são transportados a longas distâncias pelo vento, chuva etc. e se acumulam em plantas, solo e animais, causando danos à saúde humana. ${ }^{1}$ Além disso, as sobras de materiais de construções contendo cimento são dispostos no ambiente e elementos tóxicos contidos nesse material podem ser lixiviados para o solo e a água pela ação da chuva.

Isilkli et al. ${ }^{1}$ determinaram a concentração de cromo total no solo e em espécies de plantas de uma área rural exposta às emissões de uma fábrica de cimento. Esses autores determinaram também cromo no sangue e as condições de sensibilidade ao pó de cimento observada nos moradores da área rural. Foram feitas coletas de solo e de espécies de plantas em oito direções diferentes a partir da fábrica de cimento. As concentrações de cromo nas amostras de plantas e solo foram mais altas do que as concentrações encontradas nas amostras controle. Com relação à avaliação da sensibilidade dos moradores ao pó do cimento foi diagnosticada dermatite por contato. Além disso, as concentrações de cromo no sangue dos moradores da região também foram mais altas quando comparadas com aquelas do grupo controle.

O cimento pode ser definido como um pó fino, com propriedades aglomerantes, aglutinantes ou ligantes, que endurece sob a ação de água. $\mathrm{Na}$ forma de concreto, torna-se uma pedra artificial, que pode ganhar formas e volumes de acordo com a necessidade. Devido a essas características, o concreto é um material muito consumido pela humanidade. As principais matérias-primas para fabricação do cimento são calcário, argila, minério de ferro e gesso. As fontes dessas matériasprimas são diversas, por exemplo, o calcário pode ser obtido a partir de resíduos da fabricação de álcalis ou de conchas marinhas e escórias de altos fornos de indústrias siderúrgicas, que podem ser adicionadas como material argiloso. ${ }^{2} \mathrm{~A}$ presença de cromo no cimento se deve pela inserção desse elemento pela própria matéria-prima, que pode conter cromo em sua composição, e devido ao processo de manufatura do

\footnotetext{
*e-mail: wladianamatos@yahoo.com.br
}

cimento que pode também oxidar o cromo presente à sua forma mais tóxica, $\mathrm{Cr}(\mathrm{VI})$. Além disso, existem alguns produtos desenvolvidos a partir de cimentos, como o concreto pré-fabricado, aos quais são acrescentados sobras de couros de indústrias de calçados e artefatos de couro que contêm cromo devido ao processo de curtimento. ${ }^{3}$

Visto que o $\mathrm{Cr}$ (VI) está relacionado a doenças de pele, como dermatite por contato, e pode estar presente na composição do cimento, essa enfermidade pode ocorrer em trabalhadores da construção civil. Outro problema da presença de $\mathrm{Cr}(\mathrm{VI})$ em cimento é a aplicação desse produto para a construção de estruturas que serão utilizadas como reservatório de água, pois isso pode conduzir à contaminação da água pela lixiviação do $\mathrm{Cr}(\mathrm{VI})$ solúvel. Para eliminar os riscos causados pela presença de $\mathrm{Cr}(\mathrm{VI})$ em cimentos e produtos derivados do cimento, é necessário controlar a concentração dessa espécie de cromo nesse produto. Com esse propósito, uma diretriz europeia, a 2003/53/EC, implementada em janeiro de 2005, restringiu o uso de cimento e derivados desse produto que contenham, quando hidratado, mais do que $2 \mathrm{mg} \mathrm{kg}^{-1}$ de $\mathrm{Cr}(\mathrm{VI})$ solúvel. Além disso, exigem que esses materiais sejam tratados com agentes redutores, tais como sulfato ferroso, sulfato de manganês etc., para ajudar a manter a concentração de $\mathrm{Cr}(\mathrm{VI})$ no limite permitido. O período de tempo em que o agente redutor permanece efetivo deve ser especificado no rótulo do produto. ${ }^{4}$ Assim, há a necessidade de um método analítico para extrair eficientemente as espécies de $\mathrm{Cr}(\mathrm{VI})$ da matriz de cimento.

Potgieter et al..$^{5}$ e Panichev et al.$^{6,7}$ avaliaram o desempenho da extração alcalina de $\mathrm{Cr}(\mathrm{VI})$ empregando solução de $\mathrm{Na}_{2} \mathrm{CO}_{3}$ para diversos tipos de amostras sólidas, tais como solos, plantas e cimentos. Os autores propuseram o emprego de solução de $\mathrm{Na}_{2} \mathrm{CO}_{3}$ para a extração seletiva de $\mathrm{Cr}(\mathrm{VI})$ sendo a extração mais eficiente quando comparada a outros extratores, tais como água e $\mathrm{CO}_{2}$ A técnica de ETAAS - espectrometria de absorção atômica com atomização eletrotérmica - foi utilizada para as determinações de $\mathrm{Cr}(\mathrm{VI})$.

A técnica mais comum para a determinação de $\mathrm{Cr}(\mathrm{VI})$ em cimento ainda é a espectrofotometria de absorção molecular na região do visível. Scancar et al. ${ }^{4}$ compararam essa técnica com HPLC-ICP-MS (cromatografia líquida de alta eficiência acoplada à espectrometria de massa com plasma indutivamente acoplado) e FPLC-ETAAS (cromatografia líquida rápida de proteínas - espectrometria de absorção atômica com atomização eletrotérmica) para determinação de 
$\mathrm{Cr}(\mathrm{VI})$ em amostras de cimento. As diferenças entre as concentrações determinadas mediante as diferentes técnicas não excederam $10 \%$. Os autores concluíram que as técnicas estudadas geraram resultados exatos e apresentaram sensibilidade adequada para essa análise.

O propósito deste trabalho foi verificar a aplicabilidade da solução extratora $0,10 \mathrm{~mol} \mathrm{~L}^{-1}$ de $\mathrm{Na}_{2} \mathrm{CO}_{3}$ para extração de $\mathrm{Cr}(\mathrm{VI})$ em amostras de cimento e derivados de cimento produzidos no Brasil, executando a determinação por espectrofotometria de absorção molecular na região do visível, usando 1,5-difenilcarbazida como reagente cromogênico para verificar se esses produtos contêm concentrações de $\mathrm{Cr}(\mathrm{VI})$ superiores àquela estabelecida pela Comunidade Europeia. As concentrações de $\mathrm{Cr}$ (III) foram obtidas pela diferença entre os teores de cromo total, determinado por FAAS, e os teores de $\mathrm{Cr}(\mathrm{VI})$. A quantificação do teor total do elemento foi efetuada após a total decomposição das amostras por fusão.

\section{PARTE EXPERIMENTAL}

\section{Instrumentação}

Utilizou-se espectrômetro de absorção atômica com chama (FAAS) SpectrAA-640 Varian para as determinações de cromo total. A chama foi produzida a partir de ar e acetileno com vazões de 13,5 e 2,9 $\mathrm{L} \mathrm{min}^{-1}$, respectivamente. A resolução espectral foi de $0,2 \mathrm{~nm}$. As determinações de cromo foram realizadas usando lâmpada de catodo oco (Varian), sendo o comprimento de onda de $357,9 \mathrm{~nm}$ e a corrente elétrica aplicada de 7,0 mA. A correção de sinal de fundo foi feita com lâmpada de deutério.

Um espectrofotômetro de absorção molecular (UV-Vis modelo 482, FEMTO, São Paulo, BR) foi utilizado para as determinações de $\mathrm{Cr}(\mathrm{VI})$ após reação desse íon à 1,5-difenilcarbazida. O comprimento de onda empregado nas determinações de $\mathrm{Cr}(\mathrm{VI})$ foi de $545 \mathrm{~nm}$.

Uma mufla (EDG 3P-S, São Paulo, BR) foi empregada como fonte de aquecimento para a decomposição total das amostras por fusão.

As extrações de $\mathrm{Cr}(\mathrm{VI})$ nas amostras de cimento e derivados de cimento foram feitas mediante aquecimento em banho de areia e, após essa etapa, o extrato foi centrifugado utilizando-se uma centrífuga Hermele Z modelo 200 A (Labnet, Woodbridge, NJ, EUA).

\section{Reagentes e soluções}

Todos os materiais utilizados no trabalho foram descontaminados em banho de $\mathrm{HCl} 10 \%$ (v/v) por no mínimo 24 h, lavados com água destilada e com água destilada-desionizada (resistividade de $18,2 \mathrm{M} \Omega \mathrm{cm}$ ) obtida a partir de um sistema de purificação de água (Milli-Q da Millipore, Bedford, MA, EUA).

Em todos os experimentos, reagentes de grau analítico e água ultrapura foram empregados no preparo das soluções e amostras.

Uma solução estoque contendo $1000 \mathrm{mg} \mathrm{L}^{-1} \mathrm{de} \mathrm{Cr}(\mathrm{VI})$ foi preparada, dissolvendo-se 2,830 g de $\mathrm{K}_{2} \mathrm{Cr}_{2} \mathrm{O}_{7}$ da Riedel-de Haën (Alemanha), previamente seco à temperatura de $140^{\circ} \mathrm{C}$, em $1,00 \mathrm{~L}$ de água. Para a determinação de cromo total, as soluções de calibração foram obtidas a partir de diluição de solução estoque de $\mathrm{Cr}$ (III), da Merck.

Os reagentes, ácido clorídrico $36 \%$ (m/m), 1,5-difenilcarbazida, carbonato de sódio, carbonato de potássio e ácido bórico foram adquiridos da Merck (Alemanha). A acetona empregada no preparo da solução de 1,5-difenilcarbazida foi adquirida da Synth (Brasil) e o ácido sulfúrico $98 \%(\mathrm{~m} / \mathrm{m})$ para ajuste de $\mathrm{pH}$ e preparo da solução de 1,5-difenilcarbazida foi obtido da Mallinckrodt (México).

\section{Extração e determinação de $\operatorname{Cr}(\mathrm{VI})$}

Utilizou-se solução $0,10 \mathrm{~mol} \mathrm{~L}^{-1}$ de $\mathrm{Na}_{2} \mathrm{CO}_{3}$ para a extração de $\mathrm{Cr}(\mathrm{VI})$ nas amostras de cimento e derivados de cimento. A solução extratora foi preparada pesando-se 1,06 g de carbonato de sódio e dissolvendo-se o sal com água para um volume de $100 \mathrm{~mL}$.

As determinações de $\mathrm{Cr}(\mathrm{VI})$ em todas as amostras foram efetuadas empregando-se espectrofotometria de absorção molecular na região do visível, utilizando-se 1,5-difenilcarbazida como reagente cromogênico. De acordo com esse método, o $\mathrm{Cr}(\mathrm{VI})$ presente na solução reage com solução de 1,5-difenilcarbazida em meio ácido $(\mathrm{pH} 1,0)$ produzindo uma solução violeta. Esse é um método sensível e seletivo para a determinação espectrofotométrica de $\mathrm{Cr}(\mathrm{VI}){ }^{8}$

Para preparar a solução de 1,5-difenilcarbazida, 0,2 g desse reagente foi pesado e dissolvido em $100 \mathrm{~mL}$ de acetona contendo 1 $\mathrm{mL}$ de $\mathrm{H}_{2} \mathrm{SO}_{4}(1+9(\mathrm{v} / \mathrm{v}))$. A solução foi armazenada em um frasco âmbar para evitar a degradação fotoquímica do reagente. A solução de 1,5-difenilcarbazida foi peparada semanalmente.

A extração de $\mathrm{Cr}(\mathrm{VI})$ em amostras de cimento e derivados de cimento foi realizada pesando-se aproximadamente $200 \mathrm{mg}$ de amostra em béquer e a essa massa foram adicionados $10 \mathrm{~mL}$ de solução extratora $\left(0,10 \mathrm{~mol} \mathrm{~L}^{-1} \mathrm{de}_{2} \mathrm{Na}_{3}\right)$. Essa mistura foi mantida por 10 min sob ebulição em banho de areia, depois resfriada à temperatura ambiente e transferida para frasco graduado de $15 \mathrm{~mL}$, sendo o volume ajustado para $14 \mathrm{~mL}$ com água. Em seguida, a mistura foi centrifugada (4000 rpm) por $10 \mathrm{~min}$ e $10 \mathrm{~mL}$ do sobrenadante foram retirados e transferidos para outro frasco graduado de $15 \mathrm{~mL}$, onde o reagente 1,5-difenilcarbazida foi adicionado. $\mathrm{O} \mathrm{pH}$ da solução foi ajustado para 1,0 com solução de $\mathrm{H}_{2} \mathrm{SO}_{4} 5 \mathrm{~mol} \mathrm{~L}^{-1}$ e, a seguir, adicionou-se 1 $\mathrm{mL}$ de solução de 1,5-difenilcarbazida. $\mathrm{O}$ volume final da solução foi ajustado para $15 \mathrm{~mL}$ e, finalmente, a determinação quantitativa do $\mathrm{Cr}(\mathrm{VI})$ foi efetuada por espectrofotometria de absorção molecular. A curva analítica foi obtida diluindo-se a solução estoque de $\mathrm{Cr}(\mathrm{VI})$ no mesmo meio do branco. O branco é a solução resultante do procedimento de extração de $\mathrm{Cr}(\mathrm{VI})$ na ausência da amostra. Os experimentos foram realizados em triplicata.

Visando avaliar o procedimento de extração efetuou-se experimento de adição e recuperação de $\mathrm{Cr}(\mathrm{VI})$ na amostra de cimento. Para isso, adicionou-se $\mathrm{Cr}(\mathrm{VI})$ a $200 \mathrm{mg}$ da amostra de modo a se obter $50 \mu \mathrm{g} \mathrm{kg}^{-1}$ de $\mathrm{Cr}(\mathrm{VI})$. O procedimento de extração de $\mathrm{Cr}(\mathrm{VI})$ foi o mesmo anteriormente descrito para amostras de cimento.

\section{Teste de estabilidade do $\mathrm{Cr}(\mathrm{VI})$}

Avaliou-se a estabilidade da espécie $\mathrm{Cr}(\mathrm{VI})$ na solução extratora contendo $0,10 \mathrm{~mol} \mathrm{~L}^{-1} \mathrm{de}_{\mathrm{Na}_{2}} \mathrm{CO}_{3}$. Os experimentos foram realizados em triplicata.

Nesse experimento, uma solução contendo $12,5 \mathrm{mg} \mathrm{L}^{-1}$ de $\mathrm{Cr}(\mathrm{VI})$ foi preparada a partir da solução estoque de $\mathrm{Cr}(\mathrm{VI})$. Uma alíquota de $5,0 \mathrm{~mL}$ da solução com $12,5 \mathrm{mg} \mathrm{L}^{-1}$ de $\mathrm{Cr}(\mathrm{VI})$ foi adicionada a $25 \mathrm{~mL}$ da solução contendo $0,10 \mathrm{~mol} \mathrm{~L}^{-1}$ de $\mathrm{Na}_{2} \mathrm{CO}_{3}$. A mistura foi aquecida em um banho de areia por $30 \mathrm{~min}$, de maneira a simular o procedimento adotado para extração de $\mathrm{Cr}(\mathrm{VI})$ nas amostras. Após a etapa de aquecimento, a solução foi resfriada à temperatura ambiente, transferida para frasco de $50 \mathrm{~mL}$ graduado e o volume foi ajustado para $25 \mathrm{~mL}$ com água. Em seguida, uma alíquota de $2 \mathrm{~mL}$ dessa solução foi retirada para quantificação de $\mathrm{Cr}(\mathrm{VI})$. Posteriormente, adicionou-se ao frasco $\mathrm{H}_{2} \mathrm{SO}_{4} 0,1 \mathrm{~mol} \mathrm{~L}^{-1}$ visando ajustar o $\mathrm{pH}$ para 1,0 , sendo, em seguida, adicionado $1 \mathrm{~mL}$ de 1,5-difenilcarbazida. $\mathrm{O}$ volume final foi ajustado para $25 \mathrm{~mL}$ com solução de $\mathrm{H}_{2} \mathrm{SO}_{4} 0,1 \mathrm{~mol} \mathrm{~L}^{-1} \mathrm{e}$ a absorbância da solução de coloração violeta resultante foi medida.

Esse mesmo experimento foi repetido, porém, além da solução padrão de $\mathrm{Cr}(\mathrm{VI})$, solução padrão de $\mathrm{Cr}(\mathrm{III})$ também foi adicionada com o intuito de se avaliar o comportamento do cromo hexavalente na presença de cromo trivalente, observando-se as porcentagens de recuperação de $\mathrm{Cr}(\mathrm{VI})$. Assim, soluções com 12,5 $\mathrm{mg} \mathrm{L}^{-1}$ de $\mathrm{Cr}(\mathrm{III}) \mathrm{e}$ $\mathrm{Cr}(\mathrm{VI})$ foram preparadas a partir das soluções estoque com $1000 \mathrm{mg}$ 
$\mathrm{L}^{-1}$ de $\mathrm{Cr}$ (III) ou $\mathrm{Cr}$ (VI). Alíquotas de 5,0 mL de solução contendo 12,5 $\mathrm{mg} \mathrm{L}^{-1}$ de $\mathrm{Cr}$ (III) e $\mathrm{Cr}$ (VI) foram adicionadas a $25 \mathrm{~mL}$ de solução contendo $0,10 \mathrm{~mol} \mathrm{~L}^{-1}$ de $\mathrm{Na}_{2} \mathrm{CO}_{3}$. O procedimento adotado foi idêntico ao descrito no parágrafo anterior para as soluções contendo apenas $\mathrm{Cr}(\mathrm{VI})$.

\section{Preparo de amostra para quantificação do teor total de cromo}

As amostras de cimento e derivados de cimento (ceramicola, cimentcola e rejunte) foram obtidas comercialmente e decompostas por fusão. Uma massa de $100 \mathrm{mg}$ de amostra foi pesada em cadinho de platina e, em seguida, adicionou-se cerca de $600 \mathrm{mg}$ da mistura fundente $\left[\mathrm{Na}_{2} \mathrm{CO}_{3}, 30 \% ; \mathrm{K}_{2} \mathrm{CO}_{3} 30 \%\right.$ e $\left.\mathrm{H}_{3} \mathrm{BO}_{3}, 40 \%(\mathrm{~m} / \mathrm{m})\right]$. A alíquota da amostra foi colocada entre duas porções do fundente. Ou seja, inicialmente, uma massa aproximada de $300 \mathrm{mg}$ de fundente foi colocada no cadinho, seguida de $100 \mathrm{mg}$ da amostra e, finalmente, uma última fração de $300 \mathrm{mg}$ de fundente. A mistura foi primeiramente aquecida em bico de Bunsen por cerca de 5 min até a massa fundente se transformar em um líquido fluido transparente. Após resfriamento à temperatura ambiente, o cadinho contendo a amostra foi aquecido em mufla à temperatura de $1000^{\circ} \mathrm{C}$ por um intervalo de tempo aproximado de $1 \mathrm{~h}$. Como última etapa, a mistura, depois de resfriada, foi retomada em $20 \mathrm{~mL}$ de $\mathrm{HCl}$ 1:1 (v/v), com aquecimento em banho de areia até que todo o sólido fosse dissolvido. A solução obtida foi transferida para frasco graduado de $50 \mathrm{~mL}$ e o volume da solução ajustado para $50 \mathrm{~mL}$ com água. A quantificação de cromo total foi realizada por FAAS.

A decomposição das amostras de cimento foi realizada em triplicata e a curva analítica foi preparada usando soluções de referência preparadas no mesmo meio do branco, o qual consiste na solução resultante do procedimento de decomposição por fusão na ausência da amostra, porém contendo todos os reagentes utilizados no procedimento de fusão.

\section{RESULTADOS E DISCUSSÃO}

\section{Extração e determinação de $\operatorname{Cr}(\mathrm{VI})$}

A extração de $\mathrm{Cr}(\mathrm{VI})$ nas amostras sólidas foi efetuada empregando-se solução alcalina sob aquecimento. O meio alcalino foi escolhido por minimizar os riscos de redução dos íons $\mathrm{Cr}(\mathrm{VI})$ a

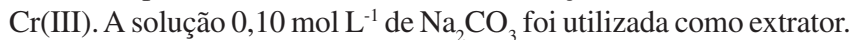
$\mathrm{O}$ aquecimento foi conduzido em banho de areia por $10 \mathrm{~min}$ a partir da ebulição da solução extratora.

A interação do íon carbonato com a superfície da amostra promove reações que extraem $\mathrm{Cr}(\mathrm{VI})$, fazendo com que compostos sólidos de $\mathrm{Cr}(\mathrm{VI})$ sejam dissolvidos e, assim, disponibilizados para medidas em fase líquida. Isso não ocorre quando se emprega apenas água para a extração de $\mathrm{Cr}(\mathrm{VI})$, pois a água se limita apenas à extração de compostos de $\mathrm{Cr}(\mathrm{VI})$ solúveis em extratores aquosos. Por exemplo, o cromo pode ser extraído do solo mediante reação entre compostos insolúveis e íons carbonato. No caso de $\mathrm{Cr}(\mathrm{VI})$ que possa existir na forma de $\mathrm{CrO}_{4}{ }^{2-}$ formando um sal insolúvel como $\mathrm{BaCrO}_{4}$, as seguintes reações são possíveis:

$$
\begin{aligned}
& \mathrm{BaCrO}_{4}+\mathrm{CO}_{3}^{2-} \leftrightarrows \mathrm{BaCO}_{3}+\mathrm{CrO}_{4}^{2-} \mathrm{K}_{\mathrm{ps}}=4 \times 10^{-2} \quad \text { (Reação global) } \\
& \mathrm{BaCrO}_{4} \leftrightarrows \mathrm{Ba}^{2+}+\mathrm{CrO}_{4}^{2-} \quad \mathrm{K}_{\mathrm{ps}}=2 \times 10^{-10} \\
& \mathrm{BaCO}_{3} \leftrightarrows \mathrm{Ba}^{2+}+\mathrm{CO}_{3}^{2-} \quad \mathrm{K}_{\mathrm{ps}}=5 \times 10^{-9} \quad \text { (Reações parciais) }
\end{aligned}
$$

A tendência da reação global é controlada pela diferença dos produtos de solubilidade dos compostos $\mathrm{BaCrO}_{4}, \mathrm{BaCO}_{3}$ e pelas concentrações de cromato e carbonato ${ }^{6}$ Considerando-se os produtos de solubilidade das duas reações parciais pode-se constatar que $\mathrm{BaCO}_{3}$ tende a se dissociar ligeiramente mais que o $\mathrm{BaCrO}_{4}$, levando o $\mathrm{Cr}(\mathrm{VI})$ na reação global a permanecer como $\mathrm{BaCrO}_{4}$. Porém, como a solução extratora é composta por $0,10 \mathrm{~mol} \mathrm{~L}^{-1}$ de $\mathrm{Na}_{2} \mathrm{CO}_{3}$, a concentração de íons carbonato no meio é elevada, o que causa o deslocamento da reação e a predominância de $\mathrm{Cr}(\mathrm{VI})$ na forma de íons $\mathrm{CrO}_{4}{ }^{2-}$, de acordo com o princípio de Le Chatelier.

\section{Estabilidade do íon $\operatorname{Cr}(\mathrm{VI})$}

A avaliação da estabilidade da espécie que se deseja quantificar é de fundamental importância na análise de especiação. No caso da especiação redox de cromo, espécies de $\mathrm{Cr}(\mathrm{VI})$ podem ser reduzidas à forma $\mathrm{Cr}(\mathrm{III})$, resultando em teores de $\mathrm{Cr}$ (VI) inferiores ao conteúdo original. Ou ainda, íons $\mathrm{Cr}$ (III) presentes no meio podem ser oxidados a $\mathrm{Cr}(\mathrm{VI})$ e, consequentemente, os teores de $\mathrm{Cr}(\mathrm{VI})$ determinados serão maiores que os reais.

Assim, o experimento de adição e recuperação de $\mathrm{Cr}(\mathrm{VI})$ na presença e na ausência de íons $\mathrm{Cr}$ (III) foi realizado usando-se solução extratora de $\mathrm{Na}_{2} \mathrm{CO}_{3} 0,10 \mathrm{~mol} \mathrm{~L}^{-1}$. No primeiro experimento, apenas $\mathrm{Cr}(\mathrm{VI})$ na concentração de $0,2 \mathrm{mg} \mathrm{L}^{-1}$ foi adicionado à solução extratora. No segundo experimento, adicionou-se $\mathrm{Cr}(\mathrm{VI})$ e $\mathrm{Cr}(\mathrm{III}) \mathrm{de}$ modo a se obter $0,2 \mathrm{mg} \mathrm{L}^{-1}$ de cada espécie na suspensão da amostra. Essas misturas foram submetidas a aquecimento em banho de areia por 30 min visando simular o procedimento de extração aplicado para as amostras.

A porcentagem de recuperação de $\mathrm{Cr}(\mathrm{VI})$ quando se adicionou apenas padrão de $\mathrm{Cr}(\mathrm{VI})$ foi $102 \pm 3$ e com adição da mistura das espécies $\mathrm{Cr}(\mathrm{VI})$ e $\mathrm{Cr}(\mathrm{III})$ foi $109 \pm 2$. Esses resultados representam os valores médios e os respectivos desvios padrão para três determinações independentes $(n=3)$.

Os dados dos testes indicam que nas condições empregadas no procedimento de preparo de amostra, isto é, aquecimento em banho de areia por $30 \mathrm{~min}$ em meio de $\mathrm{Na}_{2} \mathrm{CO}_{3} 0,10 \mathrm{~mol} \mathrm{~L}^{-1}$, não houve transformação do íon $\mathrm{Cr}(\mathrm{VI})$, tanto na ausência quanto na presença de íons $\mathrm{Cr}$ (III), pois foram obtidas recuperações de $\mathrm{Cr}$ (VI) em torno de $100 \%$. Dessa forma, confirma-se a estabilidade do $\mathrm{Cr}$ (VI) proporcionada por essa solução alcalina na extração das espécies $\mathrm{Cr}(\mathrm{III})$ e $\mathrm{Cr}(\mathrm{VI})$ em amostras sólidas. Esses resultados já eram esperados, pois, de acordo com a literatura, compostos de $\mathrm{Cr}(\mathrm{VI})$ em soluções ácidas têm forte tendência a se reduzirem. Em contrapartida, compostos de $\mathrm{Cr}(\mathrm{VI})$ têm maior estabilidade em meios com $\mathrm{pH}$ mais elevado. ${ }^{3}$

\section{Especiação de cromo em amostras de cimento e derivados de cimento}

Comumente são analisados materiais de referência certificados para avaliação da exatidão do método de análise empregado. Contudo, inexiste cimento de referência certificado e de outros materiais sólidos para análise de especiação. Dessa maneira, experimentos de adição e recuperação de $\mathrm{Cr}(\mathrm{VI})$ em amostras de cimento foram efetuados com o objetivo de avaliar, ao menos preliminarmente, a exatidão dos procedimentos de extração e determinação de $\mathrm{Cr}(\mathrm{VI})$ nessas amostras. $\mathrm{O}$ procedimento de adição de $\mathrm{Cr}(\mathrm{VI})$ foi, inicialmente, realizado antes da amostragem das replicatas mediante adição de solução de $\mathrm{Cr}(\mathrm{VI})$ ao cimento e posterior homogeneização da mistura com almofariz e pistilo. Contudo, os resultados obtidos para $\mathrm{Cr}(\mathrm{VI})$ não foram precisos. Isso indicou que a homogeneização não foi eficiente para distribuir uniformemente a espécie adicionada na amostra. A homogeneização das amostras pode ter sido dificultada pelas próprias características aglutinantes do material, visto que $\mathrm{Cr}(\mathrm{VI})$ em solução aquosa foi adicionado. Dessa maneira, optou-se por efetuar a adição de $\mathrm{Cr}(\mathrm{VI})$ após a amostragem das replicatas, ou seja, após a pesa- 
gem das massas das amostras a serem analisadas. Após a adição de $50 \mathrm{mg} \mathrm{kg}^{-1}$ de $\mathrm{Cr}(\mathrm{VI})$ apenas $31,6 \pm 1,2 \mathrm{mg} \mathrm{kg}^{-1}$ foram determinados, sendo a recuperação de $\mathrm{Cr}(\mathrm{VI})$ ao redor de $63 \%$. Os experimentos de estabilidade de $\mathrm{Cr}(\mathrm{VI})$ na solução extratora alcalina de $\mathrm{Na}_{2} \mathrm{CO}_{3}$ $0,10 \mathrm{~mol} \mathrm{~L}^{-1}$ evidenciaram que, nas condições empregadas no procedimento de extração, os íons $\mathrm{Cr}(\mathrm{VI})$ permaneceram estáveis mesmo com concentrações significativas de $\mathrm{Cr}$ (III) em solução. Dessa maneira, a baixa recuperação de $\mathrm{Cr}(\mathrm{VI})$ adicionado sob a forma de solução ao cimento, possivelmente, deve-se às interações do íon $\mathrm{Cr}(\mathrm{VI})$ com a sílica, constituinte da matéria-prima do cimento, durante o processo de hidratação do cimento, provocado pela adição de solução aquosa de $\mathrm{Cr}(\mathrm{VI})$ anteriormente à extração do $\mathrm{Cr}(\mathrm{VI})$. Provavelmente, a adição de $\mathrm{Cr}(\mathrm{VI})$ na forma de sal sólido seria o procedimento mais adequado. Esse aspecto apenas reforça a necessidade do desenvolvimento de materiais de referência com teores certificados de $\mathrm{Cr}$ (III) e $\mathrm{Cr}(\mathrm{VI})$ para diversas matrizes sólidas, possibilitando, dessa maneira, um melhor entendimento e desenvolvimento de métodos de preparo de amostra para especiação de cromo em amostras sólidas.

As concentrações de $\mathrm{Cr}(\mathrm{III})$ e $\mathrm{Cr}(\mathrm{VI})$ nas amostras de cimento e diversos derivados de cimento comerciais, tais como rejunte, cimentcola, ceramicola etc., podem ser observadas na Tabela 1 .

Tabela 1. Teores de cromo(VI) e cromo (III) em amostras de cimento e derivados de cimento

\begin{tabular}{lll}
\hline Amostra & ${ }^{1} \mathrm{Cr}(\mathrm{VI}) \mathrm{mg} \mathrm{kg}^{-1}$ & $\mathrm{Cr}(\mathrm{III}) \mathrm{mg} \mathrm{kg}^{-1}$ \\
\hline Cimento 1 & $6,8 \pm 0,2$ & $88,0 \pm 1,7$ \\
Cimento 2 & $11,3 \pm 0,4$ & $57,4 \pm 11,1$ \\
Cimento 3 & $6,1 \pm 0,2$ & $79,4 \pm 4,9$ \\
Ceramicola & $12,7 \pm 0,3$ & $57,9 \pm 0,5$ \\
Cimento 4 & $<0,01$ & $25,4 \pm 0,1$ \\
Cimento 5 & $<0,01$ & $27,1 \pm 1,2$ \\
Cimento 6 & $<0,01$ & $58,2 \pm 2,3$ \\
Cimento 7 & $<0,01$ & $47,0 \pm 0,1$ \\
Rejunte 1 & $<0,01$ & $10,9 \pm 0,4$ \\
Rejunte 2 & $<0,01$ & $58,6 \pm 0,2$ \\
Rejunte 3 & $<0,01$ & $57,0 \pm 1,2$ \\
Cimentcola & $<0,01$ & $26,5 \pm 0,2$ \\
\hline
\end{tabular}

${ }^{1}$ LOD: $0,01 \mathrm{mg} \mathrm{kg}^{-1}$. Os resultados representam os teores médios e respectivos desvios padrão $(\mathrm{n}=3)$.

De acordo com os resultados na Tabela 1, observa-se que quatro amostras, três de cimento e uma de derivado de cimento (ceramicola), continham teores de $\mathrm{Cr}(\mathrm{VI})$ acima de $2 \mathrm{mg} \mathrm{kg}^{-1}$, i.e., superiores à concentração estabelecida pela diretriz europeia. Ressalta-se que em algumas amostras as concentrações de $\mathrm{Cr}(\mathrm{VI})$ eram até seis vezes superiores ao limite permitido. Nessas amostras, o teor de $\mathrm{Cr}$ (III) foi obtido pela diferença entre as concentrações de $\mathrm{Cr}(\mathrm{VI})$ e cromo total determinadas. O teor total de cromo foi determinado após decomposição por fusão, que é um método alternativo de decomposição de materiais que não são facilmente decompostos com ácidos minerais concentrados a quente, ou que são decompostos apenas parcialmente. A fusão também é um método de preparo de amostra adequado para materiais que formam soluções ácidas instáveis, apresentando componentes com tendência para precipitação ou hidrólise como, por exemplo, a sílica.
Concentrações de $\mathrm{Cr}(\mathrm{VI})$ abaixo do limite de detecção foram observadas na maioria das amostras, indicando que o teor de $\mathrm{Cr}$ (III) é igual à concentração de cromo total. Considerando-se que a presença de $\mathrm{Cr}(\mathrm{VI})$ em cimento e derivados desse produto pode causar diversos danos ao ambiente e à saúde humana, os resultados encontrados para algumas amostras mostram a necessidade da restrição da quantidade de $\mathrm{Cr}(\mathrm{VI})$ em alguns produtos e a impossibilidade de exportação dos mesmos para países europeus. Consequentemente, a legislação brasileira também deverá implementar leis que limitem a concentração de $\mathrm{Cr}(\mathrm{VI})$ nesses produtos. Além disso, a implementação de tratamentos dos cimentos brasileiros com agentes redutores seria interessante para manter a concentração de $\mathrm{Cr}(\mathrm{VI})$ dentro do teor recomendado, evitando a oxidação do $\mathrm{Cr}$ (III) presente durante o processamento do cimento, visto que concentrações de $\mathrm{Cr}$ (III) superiores a $25 \mathrm{mg} \mathrm{kg}^{-1}$ foram encontradas nas amostras.

\section{CONCLUSÕES}

Em algumas das amostras de cimento e derivados de cimento analisadas foram encontradas concentrações de $\mathrm{Cr}(\mathrm{VI})$ superiores à concentração estabelecida pela União Europeia e, em todas as amostras, a concentração de $\mathrm{Cr}$ (III) foi maior que $10 \mathrm{mg} \mathrm{kg}^{-1}$. Dessa maneira, torna-se claro a necessidade de controle dos teores de $\mathrm{Cr}(\mathrm{VI})$ nesses produtos, caso haja interesse em viabilizá-los como produtos de exportação para a Comunidade Europeia e para evitar danos à saúde humana e ao ambiente.

A verificação da exatidão do método de quantificação do Cr(VI) foi dificultada pela provável interação do analito com a matriz do cimento durante o processo de hidratação do cimento, que ocorreu com a adição de padrão aquoso de $\mathrm{Cr}(\mathrm{VI})$ causando, dessa maneira, baixas recuperações de $\mathrm{Cr}(\mathrm{VI})$. Contudo, os dados experimentais evidenciaram que o $\mathrm{Cr}(\mathrm{VI})$ é estável no meio e nas condições de extração empregados, ou seja, nas circunstâncias utilizadas para extração de Cr(VI) não há degradação da espécie original.

\section{AGRADECIMENTOS}

Às instituições de fomento CAPES, CNPq e FAPESP pelo indispensável apoio financeiro.

\section{REFERENCIAS}

1. Isilkli, B.; Demir, T. A.; Urer, S. M.; Berber, A.; Akar, T.; Kalyoncu, C.; Environ. Res. 2003, 91, 113.

2. Associação Brasileira de Cimento Portland; http://www.abcp.org.br, acessada em Setembro 2009.

3. Baffa, I. M. P. D.; Concreto pré-fabricado com adições de resíduos de couro, PI0406251-5, 2006.

4. Scancar, J.; Milacic, R.; Séby, F.; Donard, O. F. X.; J. Anal. At. Spectrom. 2005, 20, 871 .

5. Potgieter, S. S.; Panichev, N.; Potgieter, J. H.; Panicheva, S.; Cem. Concr. Res. 2003, 33, 1589.

6. Panichev, N.; Mandiwana, K.; Foukaridis, G.; Anal. Chim. Acta 2003, 491, 81 .

7. Panichev, N.; Mandiwana, K.; Kataeva, M.; Siebert, S.; Spectrochim. Acta, Part B 2005, 60, 699.

8. Marczenko, Z.; Separation and spectrophotometric determination of elements, Ellis Horwood: Chichester, 1986. 